\title{
MRI-Based Nonrigid Motion Correction in Simultaneous PET/MRI
}

\author{
Se Young Chun ${ }^{1,3}$, Timothy G. Reese ${ }^{2,3}$, Jinsong Ouyang ${ }^{1,3}$, Bastien Guerin ${ }^{1,3}$, Ciprian Catana ${ }^{2,3}$, Xuping Zhu ${ }^{1,3}$, \\ Nathaniel M. Alpert ${ }^{1,3}$, and Georges El Fakhri ${ }^{1,3}$ \\ ${ }^{1}$ Center for Advanced Radiological Sciences, Nuclear Medicine and Molecular Imaging, Radiology Department, Massachusetts \\ General Hospital, Boston, Massachusetts; ${ }^{2}$ A.A. Martinos Center, Radiology Department, Massachusetts General Hospital, Boston, \\ Massachusetts; and ${ }^{3}$ Harvard Medical School, Boston, Massachusetts
}

Respiratory and cardiac motion is the most serious limitation to whole-body PET, resulting in spatial resolution close to $1 \mathrm{~cm}$. Furthermore, motion-induced inconsistencies in the attenuation measurements often lead to significant artifacts in the reconstructed images. Gating can remove motion artifacts at the cost of increased noise. This paper presents an approach to respiratory motion correction using simultaneous PET/MRI to demonstrate initial results in phantoms, rabbits, and nonhuman primates and discusses the prospects for clinical application. Methods: Studies with a deformable phantom, a free-breathing primate, and rabbits implanted with radioactive beads were performed with simultaneous PET/MRI. Motion fields were estimated from concurrently acquired tagged MR images using 2 B-spline nonrigid image registration methods and incorporated into a PET list-mode ordered-subsets expectation maximization algorithm. Using the measured motion fields to transform both the emission data and the attenuation data, we could use all the coincidence data to reconstruct any phase of the respiratory cycle. We compared the resulting SNR and the channelized Hotelling observer $(\mathrm{CHO})$ detection signalto-noise ratio (SNR) in the motion-corrected reconstruction with the results obtained from standard gating and uncorrected studies. Results: Motion correction virtually eliminated motion blur without reducing SNR, yielding images with SNR comparable to those obtained by gating with $5-8$ times longer acquisitions in all studies. The $\mathrm{CHO}$ study in dynamic phantoms demonstrated a significant improvement (166\%-276\%) in lesion detection SNR with MRI-based motion correction as compared with gating $(P<0.001)$. This improvement was $43 \%-92 \%$ for large motion compared with lesion detection without motion correction $(P<0.001)$. CHO SNR in the rabbit studies confirmed these results. Conclusion: Tagged MRI motion correction in simultaneous PET/MRI significantly improves lesion detection compared with respiratory gating and no motion correction while reducing radiation dose. In vivo primate and rabbit studies confirmed the improvement in PET image quality and provide the rationale for evaluation in simultaneous whole-body PET/MRI clinical studies.

\footnotetext{
Received Apr. 26, 2011; revision accepted Apr. 12, 2012.

For correspondence or reprints contact: Georges El Fakhri, Center for Advanced Radiological Sciences, Radiology/Division of Nuclear Medicine and Molecular Imaging, Massachusetts General Hospital, 55 Fruit St., Boston, MA 02114.

E-mail: elfakhri@pet.mgh.harvard.edu

Published online Jun. 28, 2012.

COPYRIGHT (C) 2012 by the Society of Nuclear Medicine and Molecular Imaging, Inc.
}

Key Words: lesion detection; motion correction; PET/MRI

J Nucl Med 2012; 53:1284-1291

DOI: 10.2967/jnumed.111.092353 he intrinsic spatial resolution of modern whole-body PET scanners is in the range of 4-7 $\mathrm{mm}$ in full width at half maximum for stationary objects $(1,2)$. However, because of the inevitable respiratory and cardiac motion, this resolution cannot be achieved in clinical imaging of the chest or abdomen, where the effective resolution becomes close to $11 \mathrm{~mm}$ (3). Therefore, many whole-body clinical PET studies may not benefit from state-of-the-art PET if motion blurring is not corrected.

Organs change location, shape, or local tissue density as they move, and complex nonrigid movement of heart muscle, lung, or abdominal organs results in image blurring (4). In such cases, coincidence data are also inconsistent with the attenuation measurement. Even if the CT-based attenuation data have negligible degradation due to movement, the attenuation correction is valid only for emission data acquired under the same stationary state as CT. Such inconsistencies between emission and attenuation data can lead to confusing artifacts (e.g., liver "banana artifacts") in the reconstructed images (5). The resulting image degradation decreases detection of lesions (6). Even when lesions are detected, their radioactivity concentration will be reduced, and apparent standardized uptake value is well below the true standardized uptake value because of motion blurring $(6,7)$.

There has been considerable research to reduce motion artifacts due to the movement of internal organs. Investigators have tried to freeze motion by exploiting the nearperiodic heart and lung motion using physiologic gating. In this context, a physiologic event such as end-inspiration (respiratory) or the QRS complex of the electrocardiogram (cardiac) is used to mark the beginning of each cycle. The respiratory cycle is typically divided into equal time bins, and data are acquired over many cycles. We will refer to these bins as timing bins to emphasize that their endpoints 
are defined relative to the gating marker. A variety of external devices (e.g., cameras, external markers, and respiratory bellows) (6), electrocardiography (8), or retrospective datadriven gating algorithms (9) have been described, but the common feature is that each timing bin includes only a small fraction of the total counts acquired, thereby reducing motion blurring at the expense of reduced signal-to-noise ratio (SNR). There are 2 strategies to improve the SNR. The most obvious and impractical is to simply increase the acquisition time to compensate for the loss of SNR. The second alternative is to use more or, better, all of the detected photons for image formation. Using sufficiently detailed and accurate information about tissue motion throughout the respiratory cycle permits formation of an image without motion blur or reduced SNR. Simple expedients, such as parameterizing the motion field assuming a rigid or affine transformation, have been used in brain PET but did not work as well for respiratory motion, and nonrigid models have been advocated $(4,10)$.

Previous attempts for nonrigid motion correction have been limited by insufficient information to effect the correction. Motion information has been derived from the same gated PET data (11), from sequentially acquired gated CT scans in PET/CT $(4,12,13)$, or from sequentially acquired MRI (14). Use of PET data to effect the correction imposes poor spatial resolution on the motion field, whereas high-resolution gated $\mathrm{CT}$ requires significant radiation exposure. Additionally, sequential PET and CT (or MRI) acquisitions do not guarantee that the same motion is captured in both modalities.

Despite these obstacles, the measured displacement field, however obtained, can be used to warp all events acquired at different times in the respiratory cycle to a reference image. In theory, the sum of all warped events yields a gated image with high SNR $(15,16)$. Alternatively, the displacement field can be incorporated into the system matrix of the reconstruction algorithm $(4,12,13,17)$. The latter has been reported to yield lower noise than summing the warped reconstructed images (18).

Applications of motion correction with simultaneous PET/MRI are beginning to appear: we have recently shown good results in PET rigid head-motion correction (19), and a motion correction method that estimates nonrigid motion from MRI and reconstructs PET data has recently been applied to locally rigid phantom studies (20).

Simultaneous PET/MRI can provide high-resolution motion information using tagged MRI because it provides natural spatial and temporal alignment between the 2 modalities and MRI can provide images with high spatial resolution and high SNR (21). Importantly, tagged MRI-based motion correction does not require additional exposure to ionizing radiation and is superior to examination of temporal changes in MRI or CT image intensity because it resolves the ambiguity associated with isointense tissues (22).

Several accurate motion estimation techniques have been developed using tagged MRI (22-24). The harmonic-phase approach yields good performance using phase information derived from the tagging patterns (24) but requires semiautomatic segmentation processing (23). In contrast, nonrigid image registration methods with data-fitting measures such as mutual information (MI) are fully automatic approaches (23).

We present and implement a new tagged MRI-based motion correction in simultaneous PET/MRI using 2 B-spline motion estimation methods. We evaluate the performance of the proposed methods for a lesion-detection task using a channelized Hotelling observer (CHO) in a deformable physical phantom and in vivo hepatic "lesions" in rabbit and primate studies. We demonstrate that our approach improves lesion detection with no additional radiation dose, and we provide the rationale for future exploration of clinical whole-body PET/MRI.

\section{MATERIALS AND METHODS}

\section{Simultaneous PET/MRI Scanner}

All studies were performed with the integrated PET/MRI scanner installed in the A.A. Martinos Center for Biomedical Imaging, Department of Radiology, Massachusetts General Hospital. This scanner consists of the BrainPET (Siemens) inserted into the bore of the 3-T Tim Magnetom Trio (Siemens) MRI scanner and allows simultaneous acquisition of MRI and list-mode PET data. The physical inner and outer diameters of the PET gantry are 36 and $60 \mathrm{~cm}$, respectively. The axial and transaxial fields of view are 19.25 and $32 \mathrm{~cm}$, respectively, and the measured spatial resolution at the center of the field of view is about $3 \mathrm{~mm}$ in full width at half maximum (25). The MRI coil consists of an inner 8-channel receive coil and a 1-channel circular polarized transmit coil with the attenuation components (e.g., capacitors) outside the field of view. For small-animal studies, the system can be configured to use the larger transmit coil in transmitreceive mode without the inner coil.

\section{Tagged MRI}

Tagged MRI imposes a spatially periodic magnetization pattern (tags) before image acquisition, using a combination of radiofrequency pulses and field gradient pulses. After the tagging pulses, the magnetization pattern persists over a specified evolution time and is distorted by motion, which can be assessed in the MR images. Spatial modulation of magnetization creates a sinusoidal modulation of magnetization in space by exciting (and subsequently dephasing) an interference pattern in the transverse magnetization due to a pair of rectangular "hard" radiofrequency pulses separated by a gradient in time (26). The magnetization pattern moves with the tissue, revealing positional changes occurring between the tagging preparation pulses and the image acquisition.

We performed tagged MRI studies with line tags for about 20 min using spatial modulation of magnetization. We modified a gradient echo sequence with spatial modulation of magnetization to permute the slice order of the acquisition according to the time after trigger to speed up the tagged MRI data acquisition using the following parameters: echo time, $2.4 \mathrm{~ms}$; repetition time, $164 \mathrm{~ms}$; flip angle, $25^{\circ}$; tagging pattern distance, $8 \mathrm{~mm}$; field of view, $128 \times$ $128 \times 128 \mathrm{~mm}$; and matrix size, $128 \times 64 \times 32$ over 3 spatialmodulation-of-magnetization axes $(x, y, z)$. A total of 32 phases (timing bins) of 3-dimensional tagged MRI data were acquired. 


\section{Deformable Motion Estimation with Tagged MRI}

We implemented B-spline nonrigid image registration with 2 different similarity measures, the sum of the squared difference (SSD) and MI $(27,28)$. For 2 images, $\rho_{S R C}$ (source image) and $\rho_{T A R}$ (target image), fields for motion (image warp) operator $T$ can be computed as:

$$
\hat{T}=\operatorname{argmin}_{T} \Psi\left(\rho_{T A R}, T \rho_{S R C}\right)+\eta R(T),
$$

where $\Psi$ is a similarity measure - SSD between $\rho_{\text {TAR }}$ and $T \rho_{S R C}$ or the negative MI, $R(T)$ is a regularizer, $\eta$ is a regularization parameter (strength), and $T$ is a motion operator that warps an image, which is parameterized by B-spline coefficients. As the number of B-spline parameters increases, one can achieve more flexible and complex nonrigid motion estimation at the expense of making the problem of nonrigid image registration underdetermined (i.e., illposed). Since the latter can have many solutions, including unrealistic motion fields, it requires regularization. Requiring the estimated motion to be invertible (i.e., the determinant of Jacobean of motion to be positive) has been shown to regularize the motion estimation problem. We used a simple regularizer that penalizes the difference of the adjacent B-spline coefficients (27). We increased a regularization parameter (the strength of the regularizer in the cost function) until all Jacobean determinant values at all voxels were positive. We applied this regularizer to both B-spline SSD and MI-based motion estimation algorithms. Cubic B-spline interpolation was used for images and estimated motion. For accurate motion description, we locate B-spline knots (coefficients) with a spacing of 4 pixels in each $x, y, z$ direction. A bilevel multiresolution scheme was used to avoid local minima. Motion fields were estimated between adjacent timing bins. B-spline interpolation was used for the composition of these motion fields to estimate motion in different timing intervals.

\section{Motion-Corrected Ordered-Subset Expectation Maximization}

The displacement fields estimated from tagged MRI were incorporated into the system matrix $a_{i j}=[A]_{i j}$ of a list-mode ordered-subsets expectation maximization (4 subsets, 10 iterations, $3 \mathrm{~mm}$ in full width at half maximum 3-dimensional gaussian postprocessing filter) algorithm to reconstruct PET images from concurrently acquired list-mode PET data $(4,13,29)$ :

$$
\rho_{i}^{(l+1)}=\frac{\rho_{i}^{(l)}}{\sum_{m=1}^{M} \Delta t_{m} \sum_{k=1}^{K} \tilde{a}_{k i}(m)} \sum_{n=1}^{N} \frac{\tilde{a}_{n i}\left(m_{n}\right)}{\sum_{j=1}^{J} \tilde{a}_{n j}\left(m_{n}\right) \rho_{j}^{(l)}+\bar{s}_{n}+\bar{r}_{n}},
$$

where $\rho$ is the estimated image in the reference frame, $m_{n}$ is the timing index corresponding to the coincidence $n$, and $l$ is the iteration number. The system matrix $\tilde{a}_{i j}(m)=[A T(m)]_{i j}$ was deformed by motion operators $T(m)$ registering the bin $m$ to the reference bin (frame). $\Delta t_{m}$ is the fraction of the acquisition time corresponding to the timing bin $m$. The MRI coil attenuation and sensitivity corrections were performed in the system matrix. Motion-dependent (subject's) attenuation correction was also performed in the system matrix by deforming the reference attenuation map to each timing bin $m$ (4). The attenuation map in the reference timing bin was obtained by segmenting MR images into 2 components: soft tissue $\left(\mu=0.096 \mathrm{~cm}^{-1}\right)$ and air $\left(\mu=0 \mathrm{~cm}^{-1}\right)$ (30). The MRI coil attenuation map was obtained from a separate CT scan and carefully aligned with PET/MRI geometry. The displacements measured after the radiofrequency coil and PET insert were repeatedly repositioned inside the scanner were smaller than $1 \mathrm{~mm}$ along and $1^{\circ}$ about all the axes. Our results demonstrate that these displacements are not expected to cause major changes in the reconstructed image (31). Random coincidence correction $\bar{r}$ was performed using a delayed coincidence window, and scatter correction $\bar{s}$ was obtained using the single scatter simulation with 1 iteration. In this paper, we assumed that $\bar{r}$ and $\bar{s}$ were not dependent on the subject's motion since scatter and random coincidences vary slowly and are essentially low-frequency functions when activity is stationary.

\section{Task-Based Performance Evaluation}

We assessed the improvement achieved by tagged MRI-based motion correction in simultaneous PET/MRI for the task of lesion detection in signal-known-exactly-background-known-exactly design using $\mathrm{CHO}$. $\mathrm{CHO}$ has been shown to yield excellent results in predicting human detection performance (32). We used 5-channel Laguerre-Gauss templates $U$ and generated $\mathrm{CHO}$ test statistics with reconstructed image $\rho$ :

$$
\lambda_{C H O}=\left[U^{T}\left(\frac{1}{2} U\left[(K)_{0}+(K)_{1}\right] U^{T}\right)^{-1} U\left((\bar{\rho})_{1}-(\bar{\rho})_{0}\right)\right]^{T} \rho
$$

where ()$_{1}$ denotes lesion present (alternative hypothesis $\left.\mathrm{H}_{1}\right),()_{0}$ denotes lesion absent (null hypothesis $\mathrm{H}_{0}$ ), and $\bar{\rho}$ and $K$ are the mean vector and ensemble noise covariance matrix, respectively, over multiple noise acquisitions (15-20 realizations). In Equation 3 , the signal $(\bar{\rho})_{1}-(\bar{\rho})_{0}$ that we would like to detect can be contaminated by motion artifacts in the absence of motion correction. To address this issue, we chose the signal from the gated approach using a long acquisition as the reference signal and replaced $(\bar{\rho})_{1}-(\bar{\rho})_{0}$ for each method by $\left(\bar{\rho}_{\text {gated }}\right)_{1}-\left(\bar{\rho}_{\text {gated }}\right)_{0}$ of the gated method. CHO SNR was computed on the basis of the test statistics as follows (32):

$$
S N R_{C H O}=\left[\frac{\left[\left(\bar{\lambda}_{C H O}\right)_{1}-\left(\bar{\lambda}_{C H O}\right)_{0}\right]^{2}}{0.5\left[\operatorname{var}\left(\lambda_{C H O}\right)_{1}+\operatorname{var}\left(\lambda_{C H O}\right)_{0}\right]}\right]^{1 / 2} \cdot \quad \text { Eq. } 4
$$

We computed CHO SNR defined in Equation 4 for 4 different image reconstruction methods: gated images, no motion correction, tagged MRI-based motion correction with SSD, and MI. We calculated $P$ values with the permutation test $(10,000$ resampling without replacement) to assess the statistical significance of the difference among the results of the 4 methods.

\section{Tagged MRI-Based Motion Correction Studies}

Phantom Study. We constructed a physical phantom that mimics respiratory and cardiac motion (Supplemental Fig. 1) consisting of viscous methyl cellulose gel with background ${ }^{18} \mathrm{~F}$-FDG activity (11.7 MBq) in which a balloon was suspended. Four 1-cm-diameter spheres with different lesion-to-background ratios $(2: 1,2.5: 1,3: 1$, $4: 1)$ were attached to various locations with different motion amplitudes and patterns. The balloon was regularly inflated and deflated $(\sim 1 \mathrm{~Hz})$ by a ventilator (Harvard Apparatus), and the intramural pressure was tracked by an amplifier with an Edwards pressure transducer (Sonometrics Corp.). The amplifier signal served as a trigger for the MRI scanner and was incorporated in the PET data stream. PET list-mode data were acquired for $120 \mathrm{~min}$ and used to generate 20 independent 6-min noise realizations. 
Rabbit Studies. We also conducted 2 in vivo rabbit studies under a protocol approved by the Massachusetts General Hospital Institutional Animal Care and Use Committee. Both animals were male New Zealand White rabbits $(3.5 \mathrm{~kg}, 14 \mathrm{wk}$ old, and $4 \mathrm{~kg}$, 15 wk old). The gating signal was obtained from respiratory bellows. Six ${ }^{18}$ F-FDG small lesions (3 beads with $148-222 \mathrm{kBq}$ for rabbit 1,3 beads with $15-56 \mathrm{kBq}$ for rabbit 2, 3- to 4-mm outer diameter) were prepared by soaking beads (Alfa Aesar) in an ${ }^{18} \mathrm{~F}-\mathrm{FDG}$ solution and implanting them in the liver and diaphragm with the animal under anesthesia. The animals were intubated endotracheally, and their respiratory rate was monitored. They were injected with $17.6 \pm 1.8 \mathrm{MBq}$ of ${ }^{18} \mathrm{~F}-\mathrm{FDG}$ intravenously. After $1 \mathrm{~h}$, simultaneous PET/MRI acquisitions were performed for $70 \mathrm{~min}$ (15 noise realizations, $\sim 5 \mathrm{~min}$ each). For rabbit 2, we injected another $237 \mathrm{MBq}$ of ${ }^{18} \mathrm{~F}-\mathrm{FDG}$ at the end of the first acquisition and scanned for another $2 \mathrm{~h}$ (study 2, $120 \mathrm{~min}$; 15 realizations, $8 \mathrm{~min}$ each) to obtain a second study with a lower lesion-to-background ratio.

Primate Study. We conducted an in vivo free-breathing primate study with the approval of the Massachusetts General Hospital Institutional Animal Care and Use Committee using the same PET/MRI setting as for the rabbit studies. The anesthetized monkey (Macaca mulatta, 5 y old, male, $4 \mathrm{~kg}$ ) was injected with $257 \mathrm{MBq}$ of ${ }^{18}$ F-FDG. After $2 \mathrm{~h}$, simultaneous PET/MRI acquisitions were performed for $30 \mathrm{~min}$ ( 5 realizations, $6 \mathrm{~min}$ each).

\section{RESULTS}

\section{Phantom Study}

MRI-based motion correction (SSD and MI) yielded reconstructed images with reduced motion artifacts and low noise compared with uncorrected or gated images. Supplemental Figure 2 shows PET phantom transaxial views reconstructed using uncorrected, gated, and MRI-based motion correction methods (SSD). The reference gated image was the mean image of gated images over 20 realizations (long gated acquisition). We selected the area with uniform radioactive gel as background and computed the contrast and SD. Lesion contrast was computed as the ratio of (sphere - background concentration) to background activity concentration, and SD was computed over noise realizations. The uncorrected method illustrates the blurring (contrast reduction) of activity in the sphere due to motion. These artifacts were greatly reduced by the gated and MRI-based motion correction methods (spheres more conspicuous and less distorted). However, the gated approach yielded increased noise, compared with MRI-based or uncorrected images, due to the reduced number of counts in each gate (12.5\% in this case). In contrast, both motion-corrected and uncorrected PET reconstructions exhibited a similar SNR, since they used the same number of detected events to form the images. MRI-based motion correction yielded image quality comparable to that of the reference gated image. Furthermore, MI and SSD yielded similar image quality.

MRI-based motion correction demonstrated significant quantitative improvement of contrast and SNR compared with gated or uncorrected methods. Table 1 shows the mean and SD of the lesion contrast-to-background ratio results for all 4 spheres over 20 realizations. Spheres 2 and 3 displayed the largest motion ( $\sim 8.8$ - and 6.5-mm amplitude, respectively), and the associated lesion contrast recovery was most apparent, up to 58\% improvement. Motion was measured with a cursor-based method using gated MR images. Spheres 1 and 4 demonstrated smaller motion ( $\sim 3.0$ - and 3.4-mm amplitude, respectively), and the associated contrast was similar in the presence or absence of motion correction, less than $13 \%$ contrast improvement. The SD of lesion contrast shows that the gating approach yielded higher variance than other methods. Table 2 shows the mean and the SD of the SNR (contrast/SD) results over the 20 noise realizations. The gated approach exhibited at least 30\% lower SNR than all other methods for large lesion-motion. For smaller motion, SNR was even lower with the gating approach because the gain in contrast became negligible compared with the increase in noise.

Tagged MRI-based motion correction improved the performance of lesion detection tasks compared with both gated and uncorrected methods. Figure 1 demonstrates this improvement for the CHO SNR: the gated method performed 59\%-72\% worse for smaller motion ranges and $44 \%-46 \%$ worse for larger motion than the uncorrected method because the increase in contrast from gating was not enough to compensate for the increase in noise. MRIbased motion correction significantly improved the $\mathrm{CHO}$ SNR by $166 \%-276 \%$ compared with the gated method and 43\%-92\% compared with the uncorrected method for large motion (spheres 2 and 3, $P<0.001$ ). For smaller motion (spheres 1 and 4), SNR improvement with SSD and MI was still statistically significant $(P<0.001)$. There was no statistical difference in lesion detection SNR associated with the SSD or MI methods.

\section{Rabbit Studies}

In vivo rabbit studies showed that MRI-based motion correction yielded improvement in image quality similar to that observed in phantom studies. Figure 2A shows the profile of 1 bead from rabbit 1 with approximately 7-mm diaphragm motion, demonstrating the motion blur and activity reduction. In Figure 2B, coronal views of the PET reconstructed images for rabbit 2 show 2 radioactive beads implanted in the liver. Even though the diaphragmatic motion of rabbit 2 in the MR image was approximately $3 \mathrm{~mm}$, we still observed an elongated shape of the bead in the superior-inferior direction (z-axis) and reduced contrast (18\% reduction). Our methods clearly improved the shape of the bead without increasing noise, compared with gating, which was comparable to the reference gated image.

Tables 1 and 2 (bottom rows) show the mean and SD of the contrast-to-background ratio and SNR for the 3 beads of rabbit $2(\sim 3-\mathrm{mm}$ diaphragm motion) over 15 realizations. We used the data from study 1 for beads 2 and 3 and the data from study 2 for bead 1 . For beads 1 and 2 (on top of the liver and on the diaphragm), we observed a $21 \%-57 \%$ contrast improvement with gating or MRI-based motion 
TABLE 1

Contrast-to-Background Ratios from 20 and 15 Noise Realizations for Phantom and Rabbit Studies, Respectively

\begin{tabular}{|c|c|c|c|c|}
\hline \multirow[b]{2}{*}{ Sphere or bead no. } & \multirow[b]{2}{*}{ Uncorrected } & \multicolumn{3}{|c|}{ MRI-based motion correction } \\
\hline & & Gated & SSD & MI \\
\hline Sphere 1 & $0.8 \pm 0.1$ & $0.9 \pm 0.2$ & $0.9 \pm 0.1$ & $0.9 \pm 0.1$ \\
\hline Sphere 2 & $1.0 \pm 0.1$ & $1.4 \pm 0.2$ & $1.3 \pm 0.1$ & $1.4 \pm 0.1$ \\
\hline Sphere 3 & $1.2 \pm 0.1$ & $1.8 \pm 0.2$ & $1.9 \pm 0.1$ & $1.8 \pm 0.1$ \\
\hline Sphere 4 & $2.1 \pm 0.1$ & $2.3 \pm 0.3$ & $2.3 \pm 0.1$ & $2.3 \pm 0.1$ \\
\hline Bead 1 & $0.7 \pm 0.1$ & $1.1 \pm 0.2$ & $1.0 \pm 0.1$ & $1.0 \pm 0.1$ \\
\hline Bead 2 & $1.4 \pm 0.2$ & $1.8 \pm 0.3$ & $1.9 \pm 0.2$ & $1.7 \pm 0.2$ \\
\hline Bead 3 & $1.5 \pm 0.2$ & $1.6 \pm 0.3$ & $1.6 \pm 0.2$ & $1.5 \pm 0.2$ \\
\hline
\end{tabular}

correction methods, but for bead 3 (inside the liver), there was less than a $6 \%$ improvement with either method because of smaller motion. MRI motion correction yielded up to $73 \%$ improvement in SNR compared with the uncorrected approach and 145\% improvement compared with the gated approach for small motion (bead 3).

Lastly, Figure $2 \mathrm{C}$ shows the CHO SNR results for rabbit 2. For motion of approximately $3 \mathrm{~mm}$, MRI-based motion correction improved CHO SNR by 19\%-33\% compared with the uncorrected approach and by 65\%-66\% compared with the gated approach for beads 1 and 2. For bead 3 (smaller motion), the improvement was not statistically significant. In this case, proposed methods achieved 134\% improved CHO SNR compared with the gated approach. SSD and MI gave similar motion estimations. Because bead 1 , being on the diaphragm, had larger displacement, the effect of motion correction was clear (statistically significant). However, bead 2 was inside the liver, the motion was relatively smaller, and the scope for improvement was reduced (higher $P$ value).

\section{Primate Study}

The primate study demonstrated that tagged MRI-based motion correction yielded PET images with visibly reduced motion artifacts and lower noise. PET coronal slices of the free-breathing monkey are shown in Figure 3A. The white arrows indicate small hot structures near the lung that ex- perienced large displacement due to their proximity to the diaphragm. The no-motion-corrected image using $6 \mathrm{~min}$ of list-mode data showed strong motion blurring near the white arrow, leading to lower contrast (0.5). However, after the use of either gating or MRI motion correction, contrast in the regions of interest was improved by $240 \%-280 \%$ (1.8-1.9). In the case of gating, this improvement was achieved at the expense of $100 \%$ greater noise than was seen on the uncorrected images. As expected, MRI motion correction yielded a level of noise similar to that of uncorrected images. Figure 3A also shows the reference gated image, which is the mean image of the gated images over 5 realizations (30 min total). Tagged MRI-based motion correction methods with a 6-min acquisition achieved quality similar to that of the 30-min gated scan in terms of motion correction and noise levels. Figure 3B shows a tagged MR image with the estimated motion fields of the respiratory motion during inspiration.

\section{DISCUSSION}

PET/MRI is an emerging technique combining both imaging modalities in a single examination. We have exploited the properties of tagged MRI acquisition to measure the motion field due to respiration and included this information in the PET reconstruction, greatly reducing the effects of motion blur. The results of our studies can be considered a proof of principle, showing that simultaneous

TABLE 2

Signal-to-Noise Ratios from 20 and 15 Noise Realizations for Phantom and Rabbit Studies, Respectively

\begin{tabular}{|c|c|c|c|c|}
\hline \multirow[b]{2}{*}{ Sphere or bead no. } & \multirow[b]{2}{*}{ Uncorrected } & \multicolumn{3}{|c|}{ MRI-based motion correction } \\
\hline & & Gated & SSD & MI \\
\hline Sphere 1 & $8.0 \pm 1.0$ & $5.2 \pm 1.1$ & $11.7 \pm 1.5$ & $11.7 \pm 1.5$ \\
\hline Sphere 2 & $11.8 \pm 1.6$ & $8.3 \pm 1.0$ & $17.7 \pm 1.8$ & $18.0 \pm 1.8$ \\
\hline Sphere 3 & $15.6 \pm 1.8$ & $10.1 \pm 1.3$ & $26.3 \pm 2.9$ & $25.5 \pm 3.0$ \\
\hline Sphere 4 & $24.2 \pm 2.4$ & $11.9 \pm 1.9$ & $29.0 \pm 4.0$ & $29.1 \pm 4.1$ \\
\hline Bead 1 & $10.0 \pm 1.9$ & $7.9 \pm 2.0$ & $15.3 \pm 3.1$ & $14.6 \pm 2.9$ \\
\hline Bead 2 & $8.5 \pm 1.2$ & $8.5 \pm 1.8$ & $14.7 \pm 1.8$ & $13.8 \pm 1.6$ \\
\hline Bead 3 & $14.2 \pm 2.0$ & $7.5 \pm 1.8$ & $18.4 \pm 4.0$ & $17.3 \pm 4.1$ \\
\hline Data are mean $\pm S D$ & & & & \\
\hline
\end{tabular}


$\square$ Uncorrected $\square$ Gated $\square$ MRI motion corrected (SSD) $\square$ MRI motion corrected (MI)

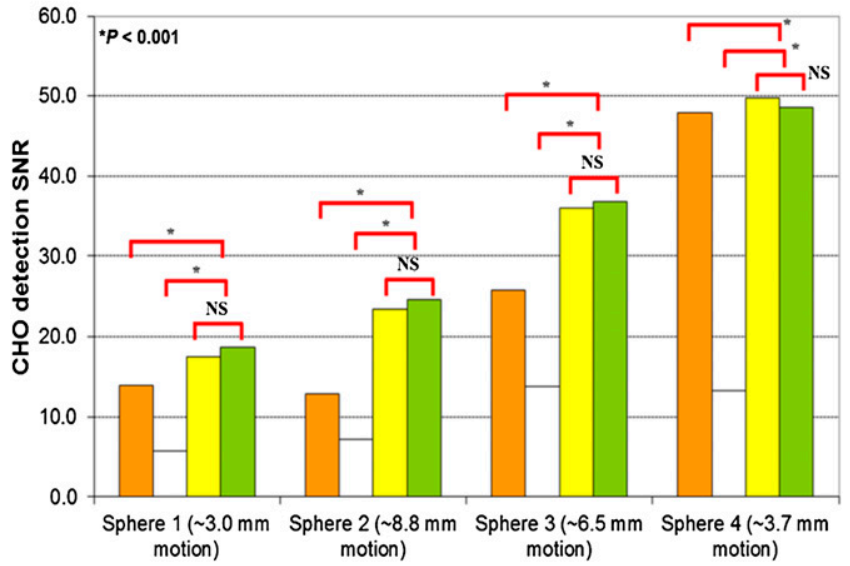

FIGURE 1. CHO SNR for 4 spheres in phantom. MRI motion correction significantly and consistently increased detection SNR for large motion in both studies. For small motion, gating yielded significant $\mathrm{CHO}$ SNR reduction as compared with no motion correction. NS = not statistically significant.

PET/MRI measurements can virtually eliminate respiratory motion blurring while improving the SNR of the reconstructed images. Though there have been many proposals for motion correction in PET and SPECT, this is the first study, to our knowledge, to demonstrate correction for nonrigid motion of phantoms and in the soft tissues of living animals in simultaneous PET/MRI. The method is general and can be extended to cardiac motion as well. Though this is the first study of its type, it suggests the possibility of clinical application, with attendant improvement in both quantitative and qualitative PET images in the abdomen of human subjects. It is important to recognize the difference between PET/MRI motion-correction methods proposed in this paper and conventional gated imaging that yields motion "freezing": both attain similar improvements in spatial resolution and contrast recovery, but conventional gating methods reject all events outside a small time window, resulting in either significant loss of SNR or long acquisition times.

Our motion correction studies were conducted on the prototype BrainPET/MRI scanner, restricting the studies to phantoms and small animals. The MRI capabilities of the prototype for tagged MRI of the torso are limited by the included MRI coil technology. As a result, acquisition of the motion field took about $20 \mathrm{~min}$ in these initial studies, compared with a few minutes with clinically available MRI coil technology. For example, current multichannel coils and multiple receivers on our recently installed mMR scanner (Siemens) enable image acceleration using SENSE (33) and GRAPPA (34). Using the spine and chest arrays would yield a 3-fold acceleration. This, in turn, will allow the acquisition of an image in $130 \mathrm{~ms}$ and the acquisition of a complete respiratory cycle of a $40 \times 20 \times 20 \mathrm{~cm}$ volume of 16 slices in less than $5 \mathrm{~min}$. This time will further decrease as sequence opti- mization, aggressive acceleration, and transverse grid tagging become available.

Our MRI-based motion correction in simultaneous PET/ MRI showed significant improvements in $\mathrm{CHO}$ detection SNR with no additional radiation dose, suggesting that corresponding improvements in clinical studies may be obtained. Proposed methods improved detection SNR dramatically with larger motion compared with studies without motion correction. Motion correction did not worsen detection SNR with smaller motion, contrary to the results obtained with the conventional gated method. Even though many lesions are easily detected with gated PET, its inherently higher noise level imposes an unfavorable tradeoff of sensitivity versus specificity. This tendency is not clear visually in some of our studies because we performed long acquisitions to achieve relatively low noise levels, but the systematically lower CHO SNR of the gated approach demonstrates this tendency quantitatively. On the basis of these data, we predict that tagged MRI-based motion correction will improve lesion detection in the clinical environment, especially for abdominal and thoracic imaging, for any patient with large or small respiratory motion. Soon, we hope to demonstrate these predictions with the recently installed mMR scanner and human observer studies with patients.

Further research is needed to fully optimize the capabilities of simultaneous PET/MRI for absolute quantitation. For example, phantom studies were not able to completely recover the original contrast in 4 hot spheres. We believe that at least part of the explanation is the lack of partialvolume correction and the use of simplified 2-class attenuation correction maps (air + soft tissue). On the other hand, even at this early stage of development, we were able to demonstrate significant improvement that was comparable to the gold standard (reference gated images) using tagged MRI motion correction methods.

MRI-based motion correction added more computational time to the PET reconstruction (e.g., ordered-subsets expectation maximization) in 2 areas. One is to estimate the motion fields between timing bins from tagged MRI, and the other is to add 1 forward and 1 transpose motion operation in the PET reconstruction algorithm. Our proofof-principle implementation was not optimized, and in studies, estimating motion between 2 timing bins took about $1 \mathrm{~h}$ using a single central processing unit; on the other hand, one can estimate all necessary motion fields in parallel for any number of timing bins. Therefore, these times can be dramatically reduced using parallel computing or graphics processing units. In list-mode orderedsubsets expectation maximization, motion correction did not increase the total PET reconstruction time significantly. In the monkey study, it took about $1 \mathrm{~h}$ per iteration to perform both motion-corrected and motion-uncorrected list-mode ordered-subsets expectation maximization on a single central processing unit, and the computation time of the motion-corrected PET reconstruction was dominated by forward and back projections, not by motion operators. 


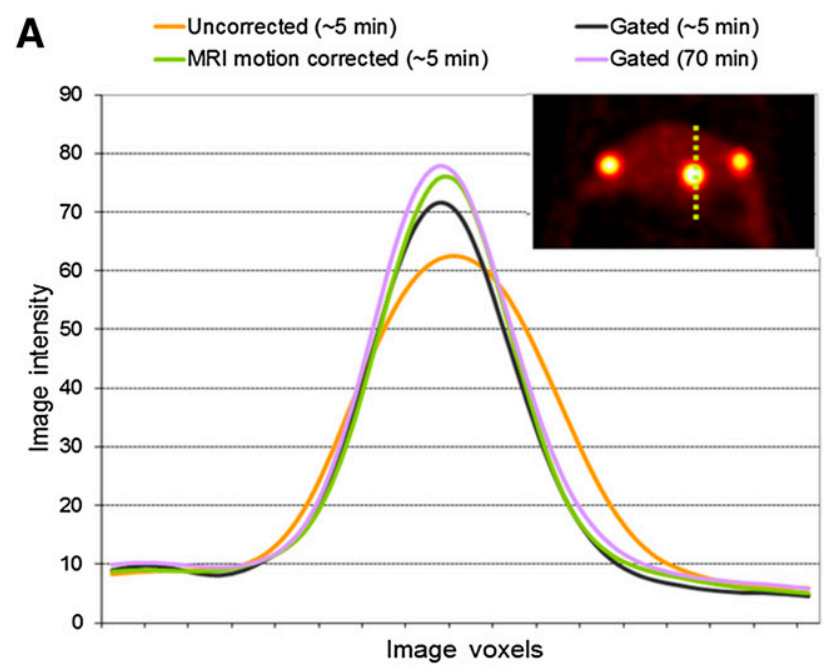

B
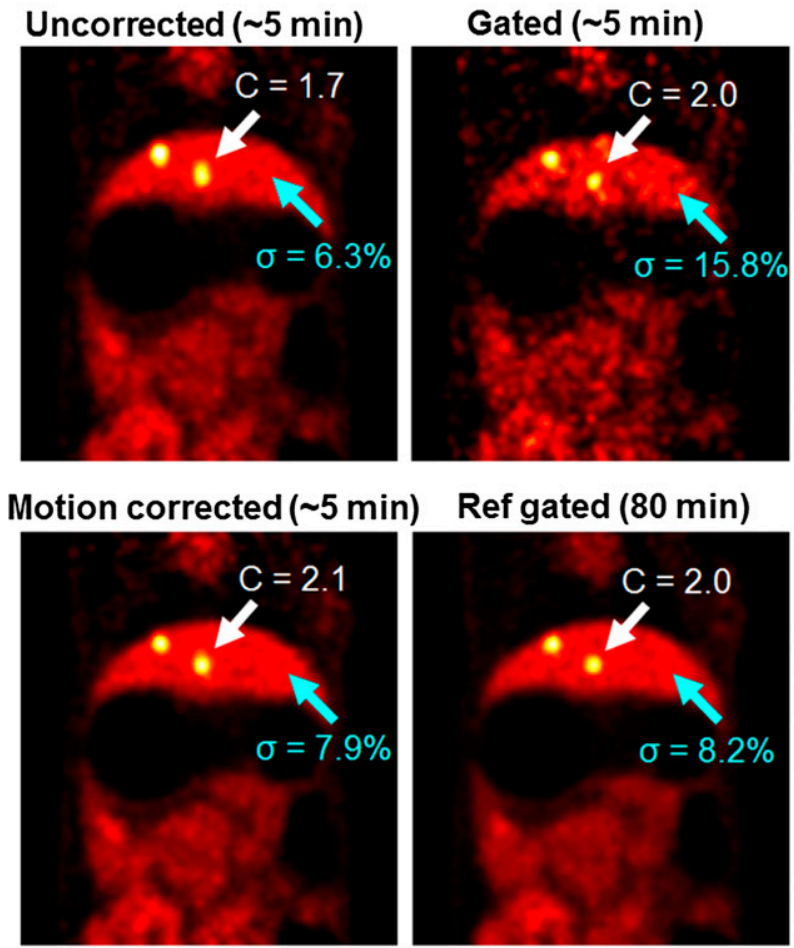

C

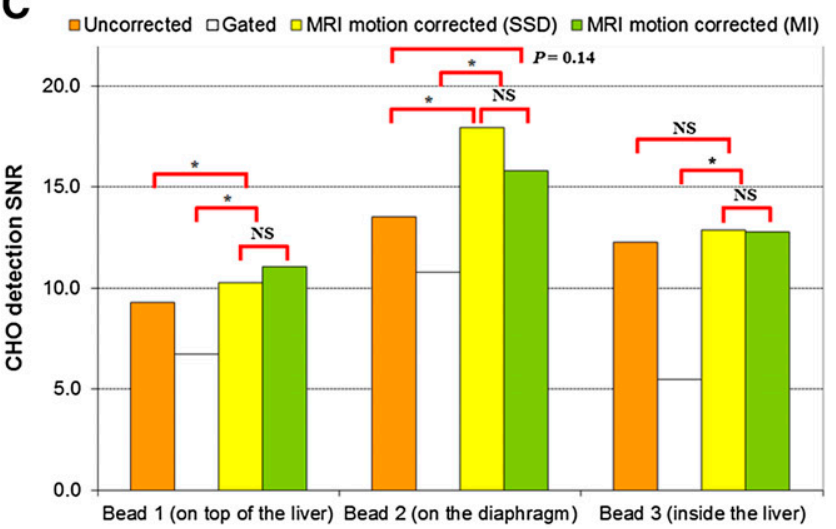

Cyclic processes such as cardiac contraction and breathing can be modeled as periodic, thus allowing the image to be formed over multiple cycles and making our task easier. Not all movement can be represented as periodic. Consider unpredictable motion, such as peristaltic motion of the gut. To image the gut, enough information for a complete image of the entire volume must be acquired within a short time, compared with the motion spectrum of the imaged object.

Lastly, higher temporal accuracy is key for accurate motion estimation. In this study, collection of tagged MR images was based on gating from respiratory bellows assuming periodic motion. However, for a long acquisition, breathing may be irregular and have a variable tidal volume. Tagged MRI interleaved with navigators has the potential to provide better motion information by tracking motion of the diaphragm directly.

\section{CONCLUSION}

To our knowledge, this is the first study to demonstrate the feasibility of in vivo MRI-based PET motion correction in simultaneous PET/MRI and the associated improvement in lesion detection. Phantom and rabbit experiments showed that MRI-based motion correction significantly improved lesion contrast, SNR, and image quality, compared with the conventional respiratory or cardiac gated approach or no motion correction. In vivo rabbit and free-breathing primate studies also clearly demonstrated an improvement of PET image quality with the proposed approaches. These results suggest improved spatial resolution and lesion detection with MRI-based motion correction in clinical studies with no additional radiation dose in recently installed simultaneous whole-body PET/MRI.

\section{DISCLOSURE STATEMENT}

The costs of publication of this article were defrayed in part by the payment of page charges. Therefore, and solely to indicate this fact, this article is hereby marked "advertisement" in accordance with 18 USC section 1734.

\section{ACKNOWLEDGMENTS}

We thank Dr. Koen Nelissen, Daniel Chonde, and Dr. Elie Moussallem for their help with the animal studies. This work was supported in part by NIH-R21-EB012326 and R01-CA165221. No other potential conflict of interest relevant to this article was reported.

FIGURE 2. (A) Profiles of reconstructed images of rabbit 1 (7-mm diaphragm motion). Significant reduction is seen with uncorrected (but not MRI-corrected) radioactivity concentration in hepatic "tumor." (B) Reconstructed PET images of rabbit 2 (3-mm diaphragm motion) with uncorrected, gated, and MRI-based motion correction methods in first scan. MRI-based motion correction methods reduced motion blur compared with uncorrected image (white arrows, $\mathrm{C}=$ contrast), without compromising noise properties as compared with gated image (blue arrows, $\sigma=\mathrm{SD}$ ). (C) CHO SNR for 3 beads in rabbit 2. MRI motion correction significantly increased detection SNR for large motion (beads near diaphragm). Ref = reference. 
A Uncorrected $(6 \mathrm{~min})$

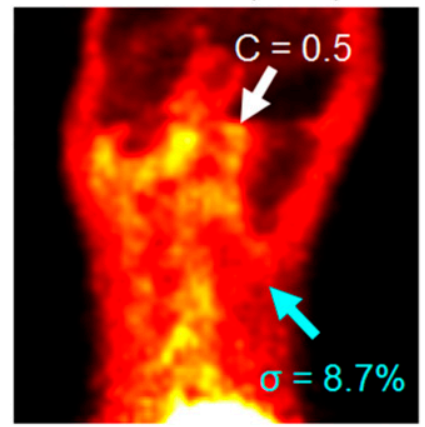

Motion corrected $(6 \mathrm{~min})$

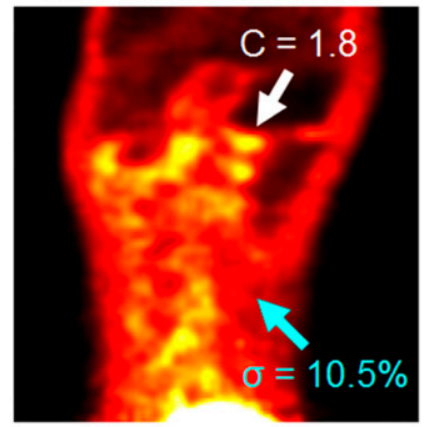

B

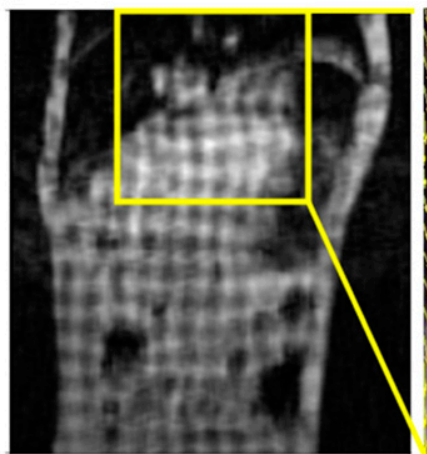

Gated (6 min)

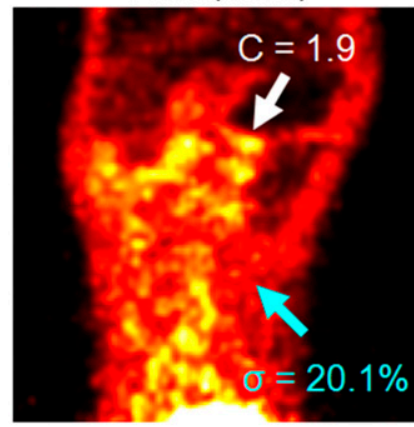

Ref gated (30 $\mathrm{min})$

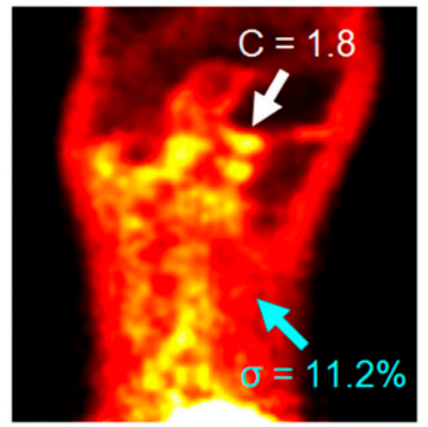

6. Nehmeh SA, Erdi YE, Ling CC, et al. Effect of respiratory gating on quantifying PET images of lung cancer. J Nucl Med. 2002;43:876-881.

7. Bundschuh RA, Martínez-Möller A, Essler M, et al. Local motion correction for lung tumours in PET/CT: first results. Eur J Nucl Med Mol Imaging. 2008; 35:1981-1988.

8. Martinez-Möller A, Zikic D, Botnar RM, et al. Dual cardiac-respiratory gated PET: implementation and results from a feasibility study. Eur J Nucl Med Mol Imaging. 2007;34:1447-1454.

9. Büther F, Dawood M, Stegger L, et al. List mode-driven cardiac and respiratory gating in PET. $J$ Nucl Med. 2009;50:674-681.

10. Montgomery AJ, Thielemans K, Mehta MA, et al. Correction of head movement on PET studies: comparison of methods. J Nucl Med. 2006;47:1936-1944.

11. Blume M, Martinez-Moller A, Keil A, et al. Joint reconstruction of image and motion in gated positron emission tomography. IEEE Trans Med Imaging. 2010;29:1892-1906.

12. Li T, Thorndyke B, Schreibmann E, Yang Y, Xing L. Model-based image reconstruction for four-dimensional PET. Med Phys. 2006;33:1288-1298.

13. Qiao F, Pan TS, Clark JWJ, Mawlawi OR. A motion-incorporated reconstruction method for gated PET studies. Phys Med Biol. 2006;51:3769-3783.

14. Reyes M, Malandain G, Koulibaly PM, et al. Model-based respiratory motion compensation for emission tomography image reconstruction. Phys Med Biol. 2007;52:3579-3600.

15. Bai W, Brady M. Regularized B-spline deformable registration for respiratory motion correction in PET images. Phys Med Biol. 2009;54:2719-2736.

16. Dawood M, Buther F, Jiang X, Schafers K. Respiratory motion correction in 3-D PET data with advanced optical flow algorithms. IEEE Trans Med Imaging. 2008;27:1164-1175.

17. Qi J, Huesman RH. List mode reconstruction for PET with motion compensation: a simulation study. eScholarship Web site. Available at: http://escholarship.org/ uc/item/4x89q5d0\#page-1. Published July 1, 2002. Accessed June 6, 2012.

18. Asma E, Manjeshwar R, Thielemans K. Theoretical comparison of motion correction techniques for PET image reconstruction. In: Nuclear Science Symposium Conference Record, 2006. Vol 3. Piscataway, NJ: IEEE. 2007: 1762-1767.

19. Catana C, Benner T, van der Kouwe A, et al. MRI-assisted PET motion correction for neurologic studies in an integrated MR-PET scanner. $\mathrm{J} \mathrm{Nucl} \mathrm{Med.}$ 2011;52:154-161.

20. Tsoumpas C, Mackewn JE, Halsted P, et al. Simultaneous PET-MR acquisition and MR-derived motion fields for correction of non-rigid motion in PET. Ann Nucl Med. 2010;24:745-750.

21. Antoch G, Bockisch A. Combined PET/MRI: a new dimension in whole-body oncology imaging? Eur J Nucl Med Mol Imaging. 2009;36:113-120.

22. Prince JL, McVeigh ER. Motion estimation from tagged MR image sequences. IEEE Trans Med Imaging. 1992;11:238-249.

23. Ledesma-Carbayo MJ, Derbyshire JA, Sampath S, et al. Unsupervised estimation of myocardial displacement from tagged MR sequences using nonrigid registration. Magn Reson Med. 2008;59:181-189.

24. Osman NF, Prince JL. Regenerating MR tagged images using harmonic phase (HARP) methods. IEEE Trans Biomed Eng. 2004;51:1428-1433.

FIGURE 3. (A) Reconstructed PET images of free-breathing monkey (7-mm diaphragm motion) with uncorrected, gated, MRI-based motion correction methods. MRI motion-correction methods significantly increased contrast compared with uncorrected image (white arrows, $\mathrm{C}=$ contrast) but did not increase noise, unlike gated image, which had increased noise (blue arrows, $\sigma=\mathrm{SD}$ ). (B) Tagged MR images with estimated motion fields (yellow box). Ref $=$ reference.

\section{REFERENCES}

1. Mawlawi O, Townsend DW. Multimodality imaging: an update on PET/CT technology. Eur J Nucl Med Mol Imaging. 2009;36:15-29.

2. Alessio AM, Stearns CW, Tong S, et al. Application and evaluation of a measured spatially variant system model for PET image reconstruction. IEEE Trans Med Imaging. 2010;29:938-949.

3. Daou D. Respiratory motion handling is mandatory to accomplish the highresolution PET destiny. Eur J Nucl Med Mol Imaging. 2008;35:1961-1970.

4. Lamare F, Ledesma-Carbayo MJ, Cresson T, et al. List-mode-based reconstruction for respiratory motion correction in PET using non-rigid body transformations. Phys Med Biol. 2007;52:5187-5204

5. Alessio AM, Kohlmyer S, Branch K, et al. CINE CT for attenuation correction in cardiac PET/CT. J Nucl Med. 2007;48:794-801.
25. Schlemmer HPW, Pichler BJ, Schmand M, et al. Simultaneous MR/PET imaging of the human brain: feasibility study. Radiology. 2008;248:1028-1035.

26. Axel L, Dougherty L. MR imaging of motion with spatial modulation of magnetization. Radiology. 1989;171:841-845.

27. Chun SY, Fessler J. A simple regularizer for B-spline nonrigid image registration that encourages local invertibility. IEEE J Sel Top Signal Process. 2009;3: $159-169$.

28. Thévenaz P, Unser M. Optimization of mutual information for multiresolution image registration. IEEE Trans Image Process. 2000;9:2083-2099.

29. Livieratos L, Stegger L, Bloomfield P, et al. Rigid-body transformation of listmode projection data for respiratory motion correction in cardiac PET. Phys Med Biol. 2005;50:3313-3322.

30. Guérin B, Reese T, Cho S, et al. Non-rigid PET motion compensation in the lower abdomen using simultaneous tagged-MRI and PET imaging. Med Phys. 2011;38:3025-3038.

31. Catana C, van der Kouwe A, Benner T, et al. Toward implementing an MRIbased PET attenuation-correction method for neurologic studies on the MR-PET brain prototype. J Nucl Med. 2010;51:1431-1438.

32. Gifford HC, King MA, De Vries DJ, Soares EJ. Channelized Hotelling and human observer correlation for lesion detection in hepatic SPECT imaging. J Nucl Med. 2000;41:514-521.

33. Pruessmann KP, Weiger M, Scheidegger MB, Boesiger P. SENSE: sensitivity encoding for fast MRI. Magn Reson Med. 1999;42:952-962.

34. Griswold MA, Jakob PM, Heidemann RM, et al. Generalized autocalibrating partially parallel acquisitions (GRAPPA). Magn Reson Med. 2002;47:1202-1210. 